\title{
Hautveränderungen im Bereich der Pfoten
}

\author{
Was gilt es zu beachten?
}

Stefanie Peters

Hautprobleme sind häufig an den Pfoten des Hundes zu finden. Die genaue Lokalisation und Kombination der Veränderungen sowie die Art und Verteilung weiterer Hautprobleme sind bei der Diagnostik ebenso zu berücksichtigen wie extrakutane Symptome. Lesen Sie, wie einzelne „Puzzlesteinchen“ Ihnen helfen, zielgerichtet zur Diagnose zu gelangen.

\section{Einleitung}

Hautprobleme an den Pfoten sind für Besitzer wie Tierarzt unschwer festzustellen. Trotzdem gilt es, nicht nur die offensichtlich veränderten Bereiche anzuschauen und sofort eine Behandlung einzuleiten, sondern eine komplette klinische und dermatologische Untersuchung durchzuführen. Schließlich manifestieren sich die wenigsten (Haut-)Erkrankungen nur im Bereich der distalen Gliedmaßen. Art und Verteilungsmuster weiterer Veränderungen, die erfahrungsgemäß vom Besitzer noch nicht bemerkt

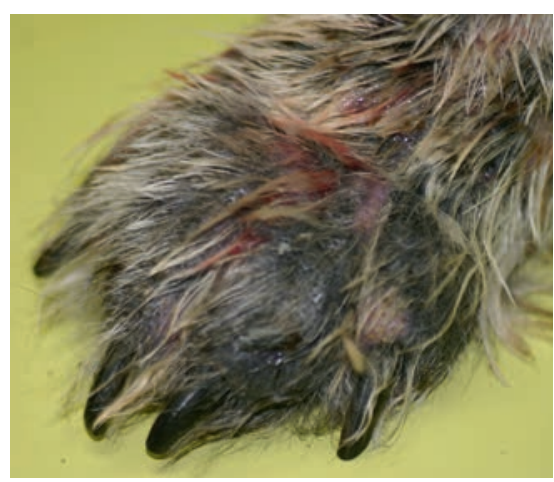

Abb. 1 Furunkulose bei einem West Highland White Terrier, der wegen seiner Allergie bereits Jahre mit Prednisolon therapiert wurde. Nach anfänglichem guten Ansprechen zeigt diese Therapie mittlerweile keinen Erfolg mehr. Die 1. Differenzialdiagnose ist eine iatrogene Demodikose mit bakterieller Sekundärinfektion. wurden, sowie weitere extrakutane Symptome helfen bei der Diagnose der Ursache erheblich. Auch auf die Informationen aus Signalement und Anamnese sollte nicht verzichtet werden.

Last but not least gilt es gerade bei den Pfoten genau hinzuschauen: Sind der verhornte Ballen, die Krallen, die Interdigitalhaut oder Kombinationen hieraus betroffen? Je nach Art und Lokalisation der Veränderungen an der Pfote kann die Liste möglicher Differenzialdiagnosen weiter eingeengt werden. Unnötige weiterführende Untersuchungen können somit vermieden werden.

\section{Interdigitale Haut}

Hautveränderungen im Bereich der interdigitalen Haut sind oft entzündlicher Natur und mit Pruritus verbunden. Dieser äußert sich in intensivem Belecken der Pfoten und daraus resultierend bei hellen Hunden eventuell in einer rostbraunen Verfärbung der Haare. Aller-

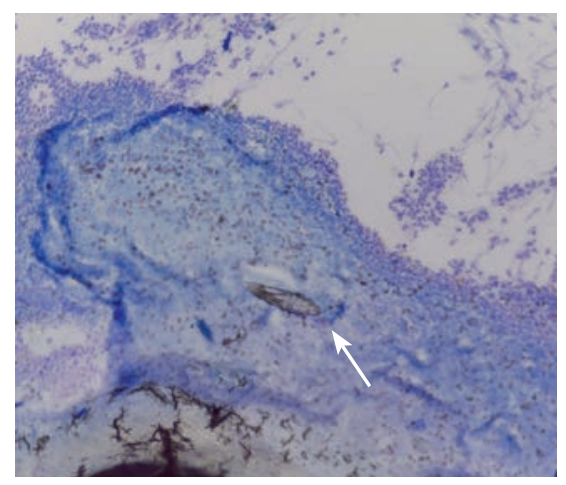

Abb. 2 Abklatschzytologie des Patienten von - Abb. 1. Eine im Diff-Quick ${ }^{\circledR}$ nicht angefärbte Larve von Demodex canis (Pfeil) ist bereits im Abklatschpräparat inmitten einer eitrigen Entzündung zu sehen. dings verbinden gerade die Besitzer kleiner Hunde das Belecken der Pfoten häufig nicht mit Juckreiz. Stattdessen interpretieren sie es als Zeichen besonderer Reinlichkeit („er putzt sich wie eine Katze“). Fragt man anamnestisch nur nach Juckreiz an den Pfoten, wird er in diesen Fällen verneint und die weitere Diagnostik womöglich in eine falsche Richtung gelenkt.

\section{Demodikose}

Bei allen Pododermatitiden sollten Untersuchungen tiefer Hautgeschabsel und evtl. Trichogramme vor allen weiteren Untersuchungen stehen. Wird eine Demodikose diagnostiziert ( Abb.1, - Abb. 2, A Abb.3), schließt sich die Frage nach der Ursache an:

- exogene Immunsuppression beispielsweise durch Kortikoidtherapie bei einem Hund mit atopischer Dermatitis

- endogene Immunsuppression bei einem mittelalten Hund infolge einer Neoplasie oder einer schweren metabolischen Veränderung

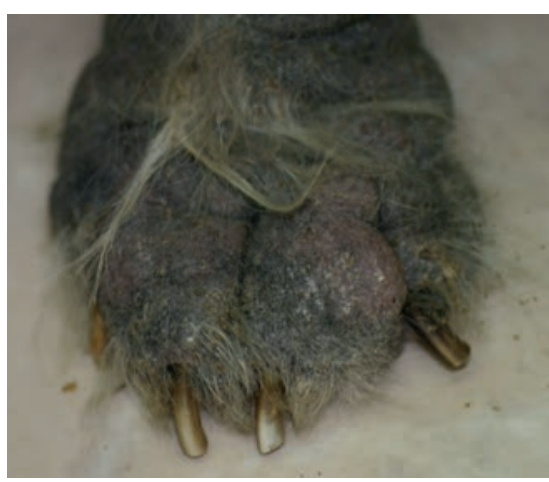

Abb. 3 Chronisch-entzündliche Veränderungen (Alopezie, Lichenifikation, Hyperpigmentierung) bei einem alten West Highland White Terrier mit sekundär bakteriell infizierter Pododemodikose. Eine exogene Immunsuppression erfolgte nicht. Als Ursache der endogenen Immunsuppression wurde 6 Monate nach Auftreten erster Hautveränderungen ein maligner Nierentumor diagnostiziert. 
- generalisierte hereditäre Demodikose beim jungen Hund

\section{.konkret}

Die 1. und wichtigste Differenzialdiagnose bei allen Pododermatitiden ist die Demodikose.

\section{Oberflächliche Sekundärinfektionen}

Bei oberflächlichen Entzündungen, die auch nässend sein können und vielfach das Krallenbett mitbetreffen, sind in den meisten Fällen Sekundärinfektionen mit Bakterien (i.d.R. Kokken) und/oder Malassezien ( $\triangleright$ Abb.4) zu finden. Diese sind in der Abklatschzytologie unschwer nachzuweisen ( $\boldsymbol{A}$ Abb.5). Neben der gezielten Therapie über mindestens 3 Wochen gilt es zu definieren, ob der Patient unter primärem Juckreiz (i.d.R. Allergien) oder sekundärem Juckreiz, z.B. durch Hypothyreose oder Demodikose,

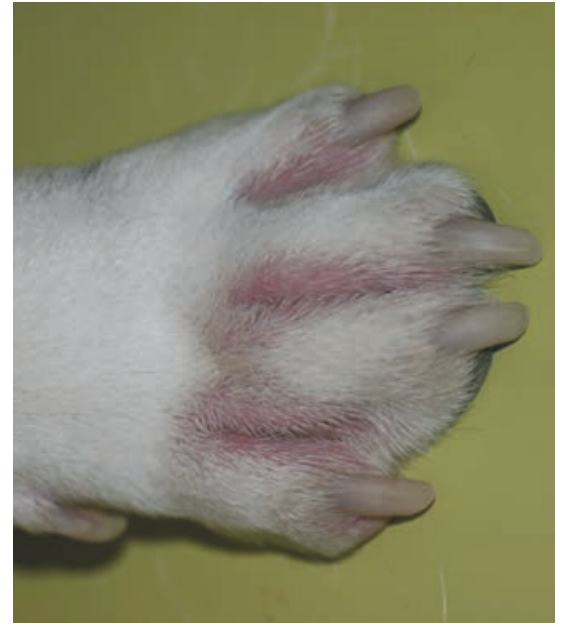

Abb. 4 Haarverlust infolge einer Selbsttraumatisierung sowie oberflächliche Entzündung der Interdigitalhaut bei einem Boxer mit atopischer Dermatitis. Zytologisch wurden Malassezien nachgewiesen ( $\bullet$ Abb.5). Die Krallen sind unverändert.

leidet. Auch hier sind andere Lokalisationen mit Art und Verteilungsmuster der Hautveränderungen ( $\triangleright$ Tab. 1$)$ sowie weitere extrakutane Symptome, z.B. bei Hy-

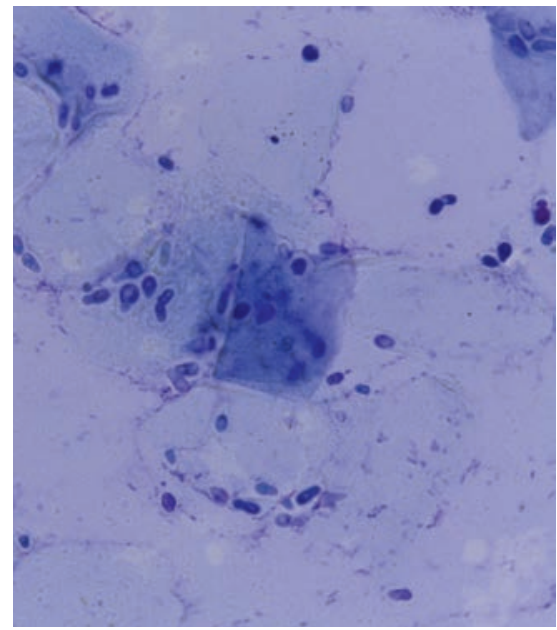

Abb. 5 Malassezien in der Abklatschzytologie beim Patienten von \ Abb. 4 (Färbung: DiffQuick $\left.{ }^{\circledR}\right)$.

pothyreose und Futterunverträglichkeit/allergie, bei der Suche nach der Primärerkrankung hilfreich.

Tab. 1 Ausgewählte Veränderungen der interdigitalen Haut.

\begin{tabular}{|c|c|c|c|c|}
\hline Primärerkrankung & $\begin{array}{l}\text { weitere Lokalisa- } \\
\text { tionen }\end{array}$ & Diagnostik & $\begin{array}{l}\text { Konta- } \\
\text { giosität }\end{array}$ & Bemerkungen \\
\hline Demodikose & $\begin{array}{l}\text { überall, wo } \\
\text { Haarfollikel sind } \\
\text { (nicht Schleimhäute, } \\
\text { Nasenspiegel, Ballen) }\end{array}$ & $\begin{array}{l}\text { tiefes Hautgeschabsel } \\
\text { evtl. Trichogramm } \\
\text { evtl. Abklatsch- } \\
\text { zytologie } \\
\text { Histopathologie }\end{array}$ & nein & $\begin{array}{l}\text { Oft nach anamnestischer Therapie mit } \\
\text { Kortikosteroiden (Allergiebehandlung) oder } \\
\text { Immunsuppressiva. } \\
\text { Erste DD bei allen Pododermatitiden. }\end{array}$ \\
\hline Malassezien-Dermatitis & $\begin{array}{l}\text { häufig Gesicht, } \\
\text { Ohren, Rutenunter- } \\
\text { seite, Hautfalten, oft } \\
\text { mit (Malassezien-) } \\
\text { Otitis }\end{array}$ & - Abklatschzytologie & nein & $\begin{array}{l}\text { Häufig sekundär bei Allergien und evtl. } \\
\text { Keratinisierungsstörungen. } \\
\text { Unbedingt gezielt therapieren und } \\
\text { Primärerkrankung suchen. }\end{array}$ \\
\hline $\begin{array}{l}\text { oberflächliche } \\
\text { bakterielle Entzündung }\end{array}$ & $\begin{array}{l}\text { wie bei Malassezien- } \\
\text { Dermatitis }\end{array}$ & - Abklatschzytologie & nein & $\begin{array}{l}\text { Nicht selten zusammen mit Malassezien- } \\
\text { Dermatitis als Sekundärinfektion. }\end{array}$ \\
\hline $\begin{array}{l}\text { Furunkulose/Zellulitis } \\
\text { (tiefe bakterielle } \\
\text { Entzündung) }\end{array}$ & $\begin{array}{l}\text { je nach Ursache wie } \\
\text { die oberflächliche } \\
\text { bakterielle Entzün- } \\
\text { dung oder auch als } \\
\text { lokalisierte tiefe } \\
\text { Entzündung nur an } \\
\text { den Pfoten }\end{array}$ & $\begin{array}{l}\text { Zytologie (Abklatsch, } \\
\text { evtl. FNA) } \\
\text { kulturelle Unter- } \\
\text { suchung mit } \\
\text { Resistenztest } \\
\text { evtl. Histopathologie } \\
\text { (Abgrenzung primär } \\
\text { steriler Prozesse) }\end{array}$ & nein & $\begin{array}{l}\text { Häufig aus immunsuppressiv therapierter, } \\
\text { oberflächlicher bakterieller Entzündung } \\
\text { entstanden. } \\
\text { Eine Demodikose muss unbedingt } \\
\text { abgeklärt werden. } \\
\text { Als interdigitale Furunkulose oft bei } \\
\text { Allergien und Hypothyreose. }\end{array}$ \\
\hline $\begin{array}{l}\text { sterile interdigitale } \\
\text { Granulome }\end{array}$ & - & $\begin{array}{l}\text { sterile Biopsie mit kul- } \\
\text { tureller Untersuchung } \\
\text { ggf. FNA }\end{array}$ & nein & $\begin{array}{l}\text { Immunsuppressive oder -modulatorische } \\
\text { Therapie erst nach Ausschluss einer } \\
\text { infektiösen Ursache/Demodikose. }\end{array}$ \\
\hline $\begin{array}{l}\text { Neoplasien (Mastzell- } \\
\text { tumor, Histiozytom, } \\
\text { Spindelzelltumor...) }\end{array}$ & je nach Neoplasie & $\begin{array}{l}\text { - FNA } \\
\text { - Histopathologie }\end{array}$ & nein & $\begin{array}{l}\text { Therapie je nach Neoplasie (exspektativ, } \\
\text { Chirurgie, Radiotherapie, Chemo- } \\
\text { therapie...). }\end{array}$ \\
\hline
\end{tabular}




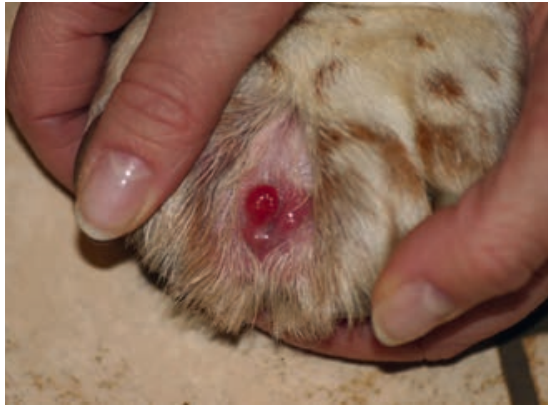

Abb. 6 Interdigitale Furunkulose bei einem Basset mit Futterunverträglichkeit/-allergie.

\section{Tiefe bakterielle Infektionen}

Tiefe bakterielle Infektionen der Haut entstehen häufig aus einer oberflächlichen Entzündung, die immunsuppressiv therapiert wurde (Anamnese), oder einer gravierenden endogenen Immunsuppression.

Bei tiefen bakteriellen Infektionen sollte zunächst eine Demodikose als Ursache abgeklärt werden.

Die Demodexmilben sind nicht selten bereits in der zytologischen Abklatschprobe zu sehen ( $\bullet$ Abb. 2 ).

Tiefe bakterielle Infektionen haben prinzipiell die gleichen möglichen Primärursachen wie die oberflächlichen Sekundärinfektionen, die abgeklärt werden sollten. Doch sind hier i.d.R. noch weitere Bakterien beteiligt, vorwiegend kleine Stäbchen (Pseudomonas spp., Proteus spp., koliforme Keime), die einer intensiven Therapie bedürfen. Neben der angesprochenen zytologischen Untersuchung sollte unbedingt eine kulturelle Untersuchung mit Resistenztest erfolgen. Es sollte eine gezielte Therapie der bakteriellen Infektion sowie der Primärursache eingeleitet werden.

\section{Interdigitale Furunkulose}

Die interdigitale Furunkulose als lokalisierte tiefe bakterielle Infektion $(\triangleright$ Abb.6) wird regelmäßig bei Allergien (v.a. Futterunverträglichkeit/-allergie) und bei Hypothyreose gesehen. Sie hat aber wie alle tiefen Pyodermien zahlreiche mögliche Primärerkrankungen. Für sie gilt prinzipiell das bereits Gesagte.

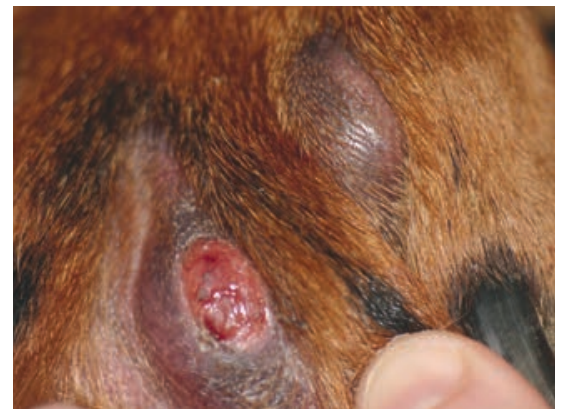

Abb. 7 Ulzerierter interdigitaler Mastzelltumor bei einem Dobermann (Differenzialdiagnose: interdigitale Furunkulose).

\section{Interdigitale Granulome}

Die Bildung primär steriler interdigitaler Granulome ist von der interdigitalen Furunkulose abzugrenzen. Die Granulome persistieren nicht selten nach abgeheilter interdigitaler Furunkulose als immunvermittelte Reaktion auf Reste von Haarfollikelteilen, freie Fettsäuren, Cholesterol etc. Sie werden dann immunmodulatorisch oder im Einzelfall auch immunsuppressiv therapiert. Ehe aber eine derartige lokale oder systemische Behandlung eingeleitet wird, müssen natürlich infektiöse Ursachen und eine Demodikose mit entsprechenden diagnostischen Verfahren ausgeschlossen sein. Dies ist mithilfe tiefer Hautgeschabsel, Trichogramm, histopathologischer und kultureller Untersuchungen steril entnommener Biopsien möglich.

\section{Neoplasien}

Auch Neoplasien wie Mastzelltumoren ( Abb.7, A Abb.8) oder Histiozytome können im Interdigitalbereich auftreten. Sie sind aber i.d.R. solitär und können mittels Zytologie und/oder Biopsie unschwer diagnostiziert werden.

\section{Ballen}

\section{Hyperkeratotische Veränderungen}

Im Bereich der Ballen werden häufig hyperkeratotische Veränderungen festgestellt. Für sie kommen insbesondere folgende Ursachen infrage:

- Pemphigus foliaceus

- hereditäre Hyperkeratose bei entsprechend prädisponierten Rassen (v.a. bei Bordeauxdogge, Kromfohrländer und Irish Terrier)

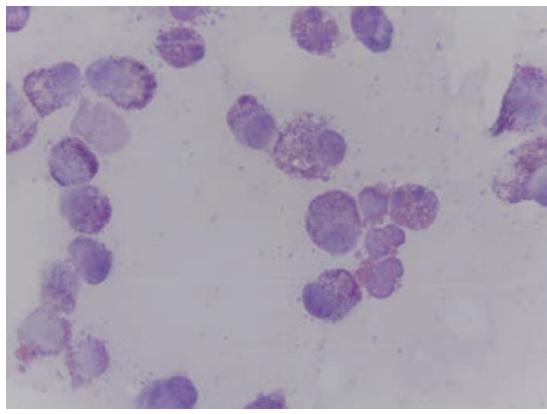

Abb. 8 Zytologie des Patienten von $>$ Abb. 7: Mastzelltumor: Mastzellen und einige eosinophile Granulozyten.

- Zink-reaktive Dermatose (Siberian Husky, Alaskan Malamute)

- Leishmaniose (bei entsprechender Anamnese)

Prädilektionsstellen beim Pemphigus foliaceus ( Abb.9) sind insbesondere Nasenrücken, eventuell Nasenspiegel, Lidbereich und die Innenseite der Pinnae, wo Pusteln mit den charakteristischen akantholytischen Zellen sowie dicke Krusten zu finden sind. Seltener ist der Inguinalbereich betroffen. Die Zink-reaktive Dermatose äußert sich zusätzlich in krustösen Veränderungen vorwiegend im Bereich der mukokutanen Übergänge, der Pinnae sowie über Druckpunkten (v.a. Ellenbogen, Sprunggelenk). Bei der familiären hereditären Hyperkeratose sind hingegen ausschließlich die Ballen betroffen, oft nur einzelne Ballen ( $\triangleright$ Abb. 10). Die Leishmaniose kann sich in zahlreichen kutanen und extrakutanen Symptomen äußern, unter anderem in einer Hyperkeratose der Ballen, häufig in Kombination mit beschleunigtem Krallenwachstum ( $\triangleright$ Abb. 11).

\section{Hepatokutanes Syndrom}

Das hepatokutane Syndrom (metabolische epidermale Nekrose) führt ebenfalls zu hochgradigen krustösen, fistelnden sowie hochschmerzhaften Ballen mit tiefen Fissuren. Diese bieten Anlass für oft wechselnde Lahmheiten ( $\bullet$ Abb. 12).

\section{konkret}

Die veränderten Ballen werden von den Besitzern häufig nicht als Lahmheitsursache erkannt. 


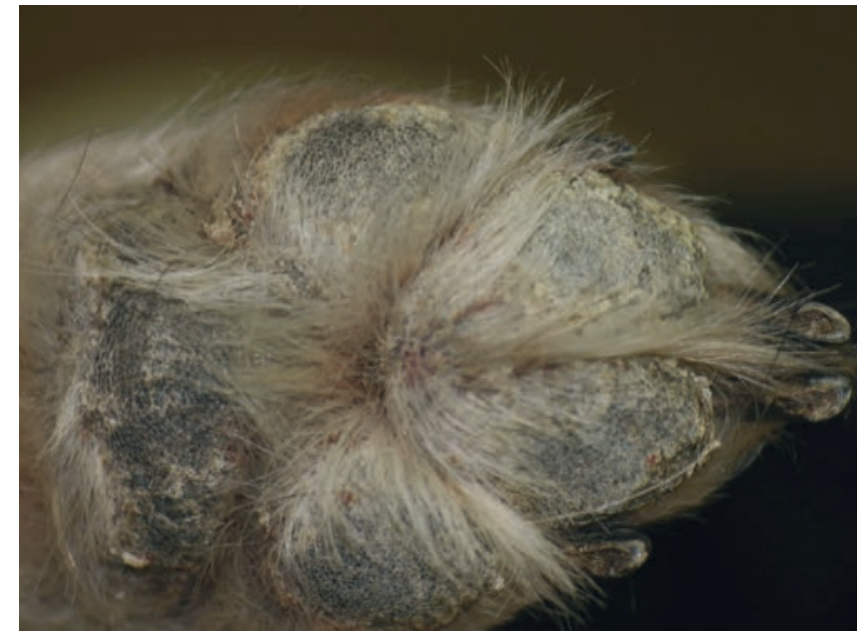

Abb. 9 Hyperkeratose der Ballen bei einem Mischling mit Pemphigus foliaceus.

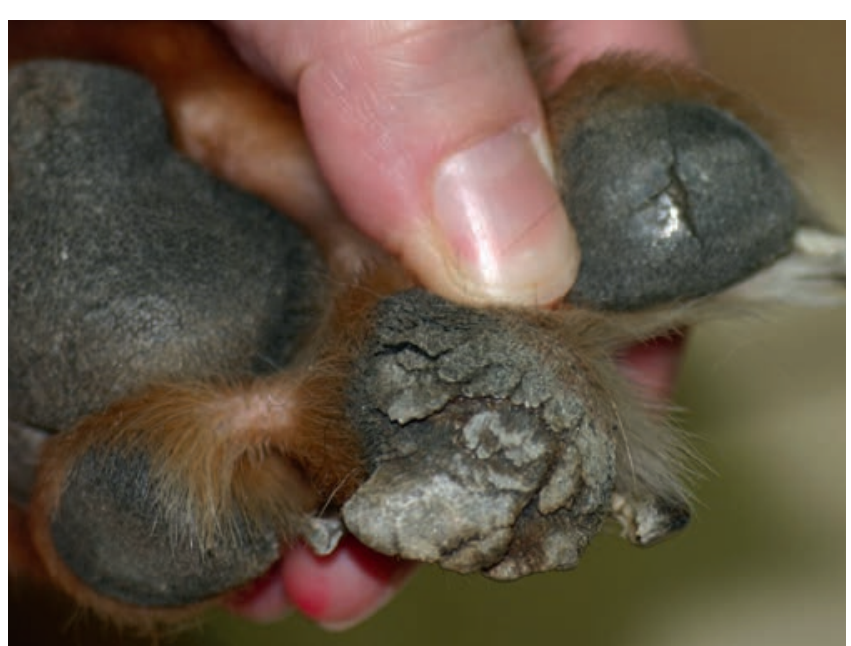

Abb. 10 Familiäre digitale Hyperkeratose bei einer 9 Monate alten Bordeauxdogge. Zu beachten sind die unterschiedlich stark veränderten Ballen an derselben Pfote.
Diese Erkrankung trifft vorwiegend bei mittelalten und alten Hunden auf, bei denen oft bereits Arthrosen diagnostiziert wurden. Diese werden zunächst für die Bewegungsstörungen verantwortlich gemacht. Weitere krustöse, erosive bis ulze- rative Hautveränderungen finden sich vor allem im Bereich der mukokutanen Übergänge und über Druckpunkten. Begleitet werden sie von meist hochgradigen Allgemeinstörungen, die häufig bereits den Hautveränderungen vorausgehen.
Das hepatokutane Syndrom ist in der Regel mit schweren Lebererkrankungen/ Leberzirrhose assoziiert, seltener mit Pankreastumoren oder Diabetes mellitus. 


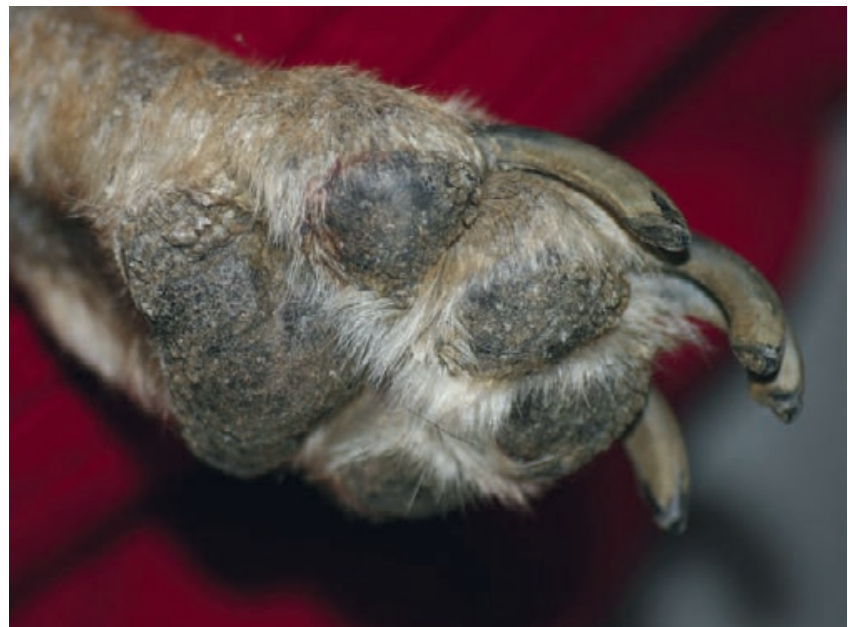

Abb. 11 Beschleunigtes Krallenwachstum und Hyperkeratose der Ballen bei einem Mischling mit Leishmaniose.

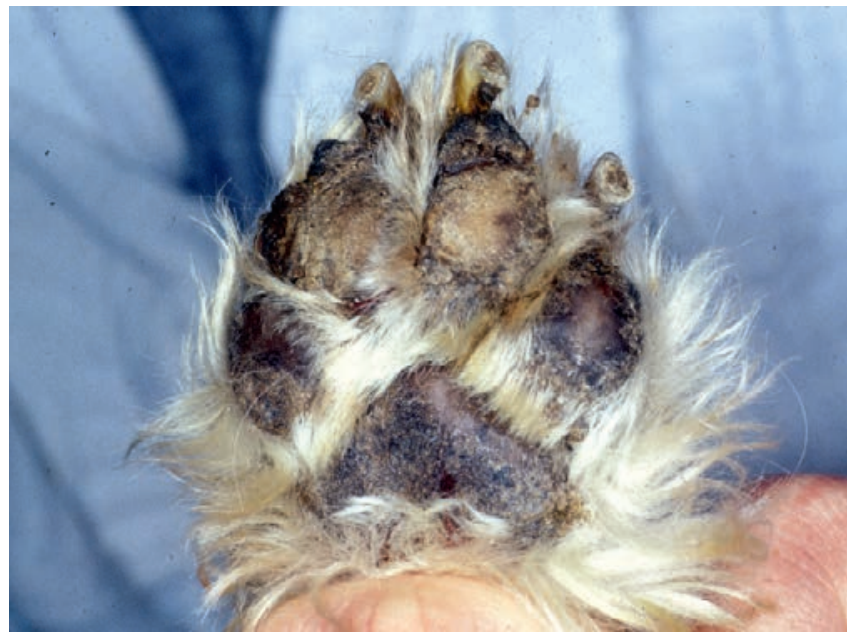

Abb. 12 Hyperkeratose und tiefe Fissuren bei einem West Highland White Terrier mit hepatokutanem Syndrom und hochgradiger Lahmheit, Anorexie und hochgradigen Allgemeinstörungen.

Tab. 2 Ausgewählte Veränderungen der Ballen.

\begin{tabular}{|c|c|c|c|c|}
\hline Primärerkrankung & $\begin{array}{l}\text { weitere Lokalisa- } \\
\text { tionen }\end{array}$ & Diagnostik & $\begin{array}{l}\text { Konta- } \\
\text { giosität }\end{array}$ & Bemerkungen \\
\hline Pemphigus foliaceus & $\begin{array}{l}\text { Gesicht (Nasen- } \\
\text { rücken, Nasenspiegel, } \\
\text { Lider), Innenseite der } \\
\text { Pinnae, evtl. } \\
\text { Inguinalbereich }\end{array}$ & $\begin{array}{l}\text { Zytologie (intakte } \\
\text { Pusteln, Innenseite der } \\
\text { Krusten) } \\
\text { - Histopathologie }\end{array}$ & nein & $\begin{array}{l}\text { Neben den spontanen gibt es auch } \\
\text { Arzneimittel-assoziierte (Anamnese) und } \\
\text { selten paraneoplastische Formen } \\
\text { (Allgemeinuntersuchung, bildgebende } \\
\text { Diagnostik). }\end{array}$ \\
\hline $\begin{array}{l}\text { hereditäre } \\
\text { Hyperkeratose }\end{array}$ & - & $\begin{array}{l}\text { - Signalement } \\
\text { - Ausschluss anderer DD } \\
\text { - evtl. familiäre Häufung }\end{array}$ & nein & $\begin{array}{l}\text { Genetisch bedingt. } \\
\text { Derzeit nicht heilbar (entsprechende } \\
\text { Beratung des Besitzers). }\end{array}$ \\
\hline $\begin{array}{l}\text { Zink-reaktive } \\
\text { Dermatose }\end{array}$ & $\begin{array}{l}\text { mukokutane } \\
\text { Übergänge, } \\
\text { Druckpunkte }\end{array}$ & $\begin{array}{l}\text { - Signalement } \\
\text { - Ausschluss anderer DD } \\
\text { - Histopathologie }\end{array}$ & nein & $\begin{array}{l}\text { Messung des Zinkspiegels im Blut oft nicht } \\
\text { diagnostisch. } \\
\text { Unbedingt nach anderen Ursachen für eine } \\
\text { verminderte enterale Zinkresorption } \\
\text { suchen. }\end{array}$ \\
\hline $\begin{array}{l}\text { hepatokutanes } \\
\text { Syndrom }\end{array}$ & $\begin{array}{l}\text { mukokutane } \\
\text { Übergänge, } \\
\text { Druckpunkte }\end{array}$ & $\begin{array}{l}\text { - klinisches Bild } \\
\text { - Biochemie } \\
\text { - bildgebende Verfahren } \\
\text { - Histopathologie }\end{array}$ & nein & $\begin{array}{l}\text { Häufig tiefe Fissuren, hochschmerzhafte } \\
\text { Pfoten. } \\
\text { Meist mit wechselnder Lahmheit und } \\
\text { hochgradigen Allgemeinstörungen. }\end{array}$ \\
\hline Vaskulitis & $\begin{array}{l}\text { je nach Ursache rein } \\
\text { kutan (kutane } \\
\text { Vaskulitis des Jack } \\
\text { Russel Terriers: } \\
\text { Nasenspiegel, } \\
\text { Ohrrand und -spitze, } \\
\text { Rutenspitze, Krallen, } \\
\text { evtl. Gaumen) oder } \\
\text { mit Symptomen } \\
\text { anderer Organsyste- } \\
\text { me (Leishmaniose, } \\
\text { SLE, Dermatomyo- } \\
\text { sitis) }\end{array}$ & $\begin{array}{l}\text { - klinisches Bild } \\
\text { - evtl. Histopathologie }\end{array}$ & nein & $\begin{array}{l}\text { Klinische Veränderungen infolge Ischämie } \\
\text { sind charakteristisch (runde, wie aus- } \\
\text { gestanzt wirkende Defekte). } \\
\text { Die Histopathologie kann i.d. R. den } \\
\text { Auslöser nicht sicher differenzieren. } \\
\text { Bei SLE und Leishmaniose weitere kutane } \\
\text { und extrakutane Symptome. } \\
\text { Bei Dermatomyositis evtl. muskuläre } \\
\text { Symptome (Megaösophagus, Bewegungs- } \\
\text { störungen). }\end{array}$ \\
\hline „Split Paw Pad Disease“ & - & $\begin{array}{l}\text { Signalement (junge, } \\
\text { adulte Hunde) } \\
\text { Histopathologie }\end{array}$ & nein & $\begin{array}{l}\text { Meist assoziiert mit Lahmheit unterschied- } \\
\text { lichen Grades. } \\
\text { Symptomatische Therapie (Entfernen des } \\
\text { abgelösten Hornes, Verbände, Analgetika, } \\
\text { ggf. Therapie von Sekundärinfektionen). } \\
\text { Präventiv: Pfotenschuhen. }\end{array}$ \\
\hline
\end{tabular}

DD: Differenzialdiagnosen, SLE: systemischer Lupus erythematodes 
Die Diagnose kann mithilfe folgender Untersuchungen unschwer gesichert werden:

- Biochemie (erhöhte Transaminasen, erniedrigtes Gesamteiweiß und erniedrigte Aminosäuren)

- abdominale Sonografie („SchweizerKäse“-Veränderungen der Leber bzw. Pankreastumor)

- charakteristische histopathologische Befunde von Hautbiopsien („umgekehrte französische Flagge“)

\section{Vaskulitiden}

Vaskulitiden führen über Entzündungen im Bereich kleiner Gefäße und eine Ischämie der nachgeordneten Gewebsbereiche im Bereich der Ballen klinisch zu meist multiplen, wie ausgestanzt wirkenden Veränderungen. Bei der kutanen Vaskulitis des Jack Russel Terriers sind auch andere charakteristische Bereiche wie Gesicht, Nasenspiegel, Ohrränder und Krallen betroffen ( $\triangleright$ Abb. 13).

Bei einer immunvermittelten Vaskulitis infolge einer Leishmaniose, einer familiären Dermatomyositis oder eines systemischen Lupus ery thematodes (SLE) sind neben den geschilderten übrigen Lokalisationen andere Organsysteme mitbetroffen. Dies erleichert die Diagnose $(\triangleright$ Tab.2).

\section{„Split Paw Pad Disease“}

Bei der zumindest selten diagnostizierten „Split Paw Pad Disease“ kommt es zu Ablösungen des Ballenhorns mit anschließenden Sekundärinfektionen und Schmerzen. Betroffen sind vorwiegend junge adulte Hunde verschiedener Rassen, bei denen sukzessive mehrere Ballen an unterschiedlichen Gliedmaßen die genannten Veränderungen entwickeln. Die Pathogenese ist nicht geklärt. Vermutet wird ein kongenitaler Defekt in der Kornifikation. Dieser schwächt das Epithel und führt letztlich in Bereichen besonderer Belastung zu einer Ablösung oberflächlicher Schichten der Epidermis von tieferen ( $\mathbf{A b b}$. 14). Klinisch zeigen sich neben den geschilderten Ablösungen oberflächlicher Epidermisbereiche in chronischen Fällen auch Veränderungen der Architektur des Ballens sowie krustöse und teilweise ulzerative Läsionen. Die Diagnose erfolgt histopathologisch.
Eine spezifische Therapie ist nicht bekannt.

\section{Krallen \\ Dermatophyten}

Veränderungen der Krallen werden erfahrungsgemäß häufig als Pilzinfektionen fehldiagnostiziert und -therapiert. Onychomykosen werden beim Hund extrem selten gesehen. Sie werden meist durch Trichophyton mentagrophytes hervorgerufen. Dieser Dermatophyt befällt keratinisiertes Gewebe (Krallen, Epidermis und Haare).

\section{konkret}

Trichophyton mentagrophytes ist kontagiös und ein Zoonoseerreger

(Anamnese).

Die Veränderungen sind nicht nur an den Krallen zu erwarten, sondern auch an anderen Lokalisationen sowie bei Kontaktpersonen und -tieren. Betroffen sind insbesondere Menschen oder Tiere, die immunsupprimiert oder noch nicht oder nicht mehr voll immunkompetent sind (sehr junge und alte Menschen bzw. Tiere). 


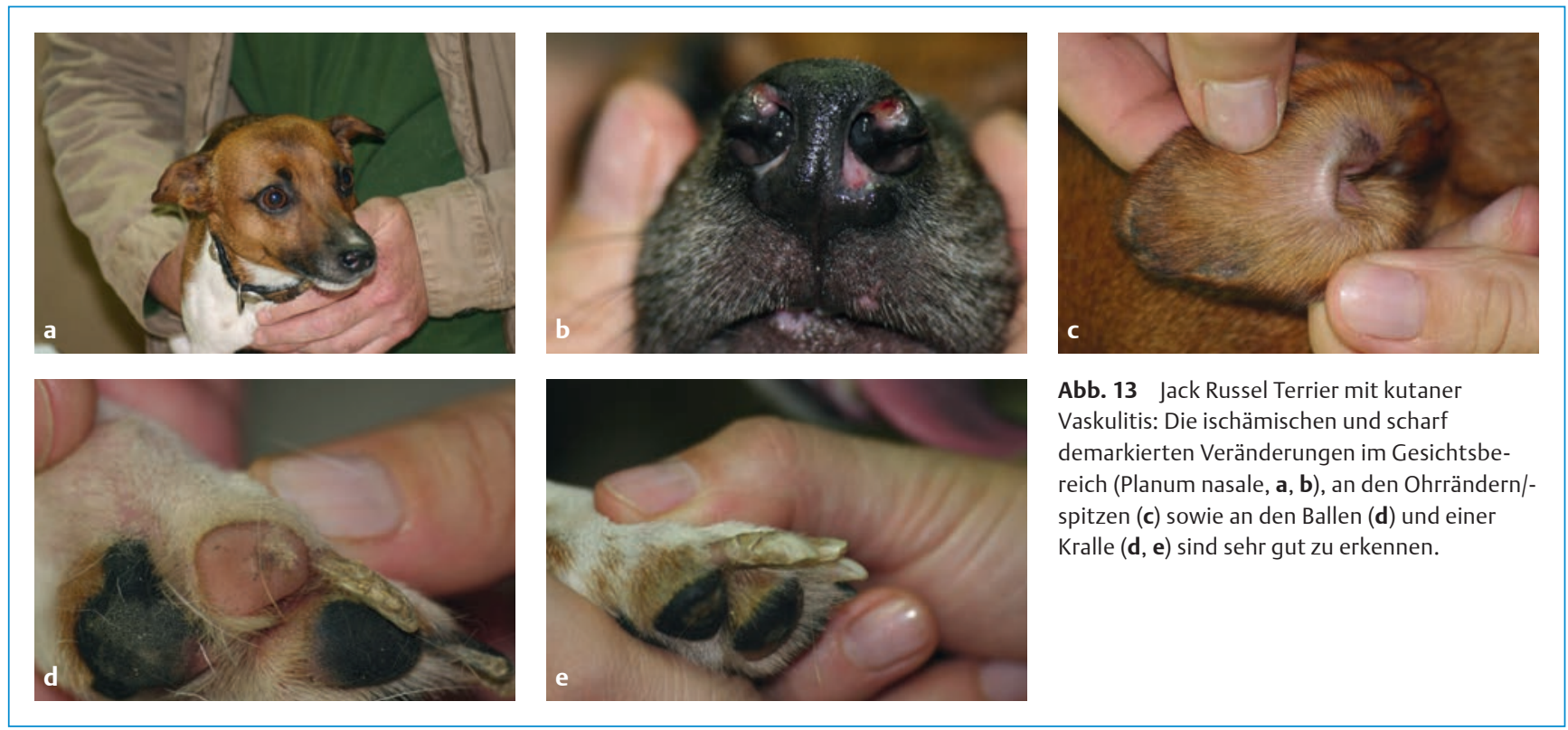

\section{Lupoide Onychodystrophie}

Sind sämtliche Krallen an allen Pfoten über einen Zeitraum von einigen Wochen oder Monaten betroffen und zeigen Veränderungen in der Qualität, Abbrechen, Spalten und letzten Endes Verlust des Krallenhorns ohne weitere betroffene Lokalisationen oder Kontagiosität, liegt eher der Verdacht auf eine lupoide Onychodystrophie ( $\vee$ Abb. 15) nahe. Hier kommt es zu immunvermittelten Reaktionen im Bereich der Grenzzone zwischen Epidermis und Dermis. Verlorengegangene Krallen wachsen nach, allerdings nicht mehr in der normalen Qualität. Sie sind prädisponiert für erneute Schädigungen. Die Sicherung der Verdachtsdiagnose erfolgt histopathologisch - hierzu empfiehlt sich die komplette Amputation des Zehenendglieds.

\section{Praxistipp \\ Der Amputation des Zehenendglieds, die im Rahmen der Diagnostik der lupoiden Onychodystrophie sinnvoll ist, stehen viele Besitzer ablehnend gegenüber. Bewährt hat sich die Amputation der Daumenkralle für die histopathologische Untersuchung. Sie ist in der Regel mitbetroffen, und erfahrungsgemäß ist die Kooperations- bereitschaft der Tierhalter bei dieser Lokalisation größer.}

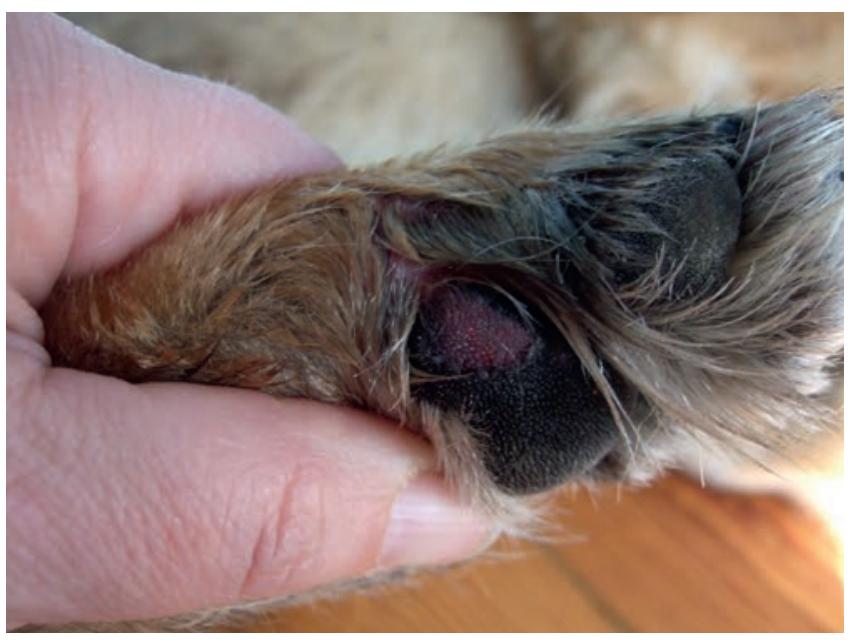

Abb. 14 „Split Paw Pad Disease“ bei einem 2-jährigen Schäferhundmischling: Das abgelöste Ballenhorn verbunden mit deutlicher Lahmheit ist gut zu erkennen. Bei diesem Patienten treten derartige Veränderungen an mehreren Zehen- und Sohlenballen auf.

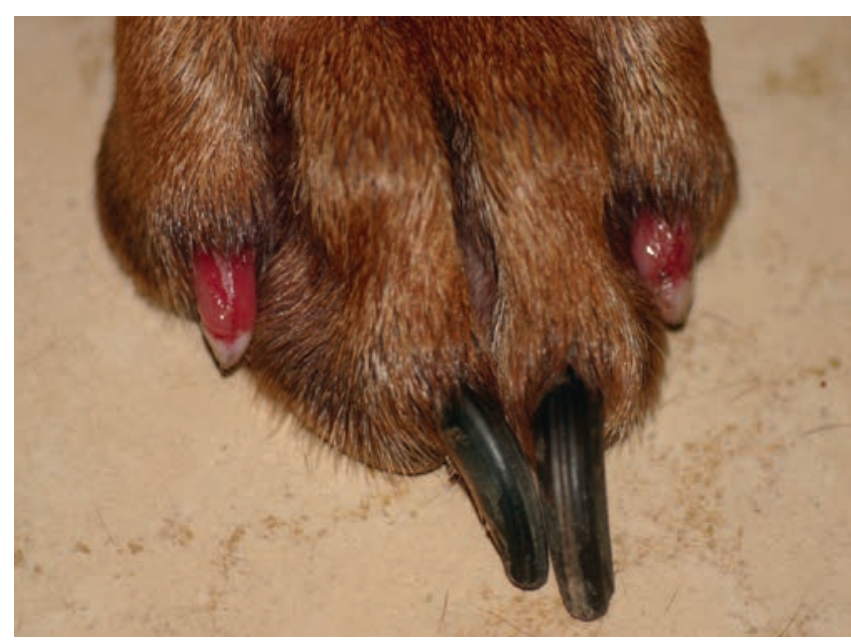

Abb. 15 Spontaner Krallenverlust infolge lupoider Onychodystrophie bei einem Rhodesian Ridgeback, einer der prädisponierten Rassen. Bei diesem Patienten waren binnen weniger Monate sämtliche Krallen an allen Pfoten betroffen. 


\section{hund.dermatologie}

Tab. 3 Ausgewählte Veränderungen der Krallen.

\begin{tabular}{|c|c|c|c|c|}
\hline Primärerkrankung & $\begin{array}{l}\text { weitere Lokalisa- } \\
\text { tionen }\end{array}$ & Diagnostik & $\begin{array}{l}\text { Konta- } \\
\text { giosität }\end{array}$ & Bemerkungen \\
\hline Onychomykose & $\begin{array}{l}\text { andere keratinisierte } \\
\text { Gewebe }\end{array}$ & $\begin{array}{l}\text { - Kultur } \\
\text { - Histopathologie (mit } \\
\text { Spezialfärbung) }\end{array}$ & ja & $\begin{array}{l}\text { Seltene Erkrankung, kommt vorwiegend bei } \\
\text { immunsupprimierten Tieren vor. } \\
\text { Häufig wird eine lupoide Onychodystrophie } \\
\text { als Onychomykose fehlinterpretiert. } \\
\text { Vorsicht bei der Interpretation kultureller } \\
\text { Untersuchungen von Krallenhorn (viele } \\
\text { angezüchtete Keime haben keine ursäch- } \\
\text { liche Beteiligung am Krankheitsprozess). }\end{array}$ \\
\hline $\begin{array}{l}\text { lupoide } \\
\text { Onychodystrophie }\end{array}$ & - & $\begin{array}{l}\text { - Ausschluss anderer DD } \\
\text { - Histopathologie }\end{array}$ & nein & $\begin{array}{l}\text { Alle Krallen an allen Pfoten sind i.d.R. } \\
\text { betroffen. } \\
\text { Die kulturelle oder histopathologische } \\
\text { Befundung abgelöster Krallen ist nicht } \\
\text { diagnostisch. } \\
\text { Für die Histopathologie empfiehlt sich die } \\
\text { Amputation eines Zehenglieds. }\end{array}$ \\
\hline $\begin{array}{l}\text { Vaskulitis (auch SLE, } \\
\text { familiäre Dermato- } \\
\text { myositis, Leishmaniose) }\end{array}$ & $\begin{array}{l}\text { je nach Ursache rein } \\
\text { kutan (kutane } \\
\text { Vaskulitis des Jack } \\
\text { Russel Terriers: } \\
\text { Nasenspiegel, } \\
\text { Ohrrand und -spitze, } \\
\text { Rutenspitze, Krallen, } \\
\text { evtl. Gaumen) oder } \\
\text { mit Symptomen } \\
\text { anderer Organsyste- } \\
\text { me (Leishmaniose, } \\
\text { SLE, Dermatomyo- } \\
\text { sitis) }\end{array}$ & $\begin{array}{l}\text { - klinisches Bild } \\
\text { - evtl. Histopathologie }\end{array}$ & nein & $\begin{array}{l}\text { Dystrophie, Deformation, Abbrechen, } \\
\text { Splittern etc. - oft nur einiger Krallen. } \\
\text { Bei SLE und Leishmaniose weitere kutane } \\
\text { und extrakutane Symptome. } \\
\text { Bei Dermatomyositis evtl. muskuläre } \\
\text { Symptome (Megaösophagus, Bewegungs- } \\
\text { störungen). }\end{array}$ \\
\hline Trauma & - & $\begin{array}{l}\text { - Anamnese } \\
\text { - klinisches Bild }\end{array}$ & nein & $\begin{array}{l}\text { Häufigste Ursache für Krallenveränderun- } \\
\text { gen (insbesondere, wenn nur } 1 \text { Zehe } \\
\text { betroffen ist). }\end{array}$ \\
\hline Neoplasien & je nach Neoplasie & $\begin{array}{l}\text { - Signalement } \\
\text { - klinisches Bild } \\
\text { - Zytologie } \\
\text { - Histopathologie } \\
\text { - evtl. Röntgenuntersu- } \\
\text { chung }\end{array}$ & nein & $\begin{array}{l}\text { Oft Prädispositionen für Rasse und Farbe } \\
\text { (unguales Plattenepithelkarzinom, malignes } \\
\text { Melanom). } \\
\text { Neoplasien im Bereich des Krallenbetts oft } \\
\text { erst auf den 2. Blick erkennbar. } \\
\text { Bei Veränderungen einer einzelnen Kralle, } \\
\text { wenn anamnestisch ein Trauma ausge- } \\
\text { schlossen werden kann, sollte sorgfältig auf } \\
\text { eine Neoplasie untersucht werden. }\end{array}$ \\
\hline
\end{tabular}

DD: Differenzialdiagnosen, SLE: systemischer Lupus erythematodes 


\section{Vaskulitis}

Auch bei der Vaskulitis werden regelmäßig Krallenveränderungen gesehen. Dystrophische, spröde, abbrechende oder deformierte Krallen infolge der Ischämie sind keine Seltenheit. Allerdings sind nicht selten nur einzelne Krallen betroffen ( $\triangleright$ Abb. 13). Vaskulitis-Symptome an den übrigen Prädilektionsstellen (Ballen, Ohrmuschel/-rand, Rutenspitze, evtl. auch Gaumen) sind zu erwarten ( $\bullet$ Tab.3). Die Abklärung der Ursache sollte unbedingt angestrebt werden, allerdings gelten etwa 50\% der Fälle als idiopathisch.

\section{Trauma}

Ein Trauma ist die häufigste Ursache für Veränderungen einzelner Krallen beim Hund (Anamnese).

\section{Neoplasien}

Bei prädisponierten Rassen und Farben (schwarze Schnauzer, Labradore, Afghanen etc.) sollte man unbedingt auch bei Veränderungen einzelner Krallen an Neoplasien denken, v.a. an unguale Plattenepithelkarzinome und maligne Melanome.
Online zu finden unter

http://dx.doi.org/10.1055/s-0035-1550101

Alle Abbildungen: @ S. Peters

\section{Literatur}

Literatur bei der Autorin.

\section{Dr. Stefanie Peters}

Tierärztliche Klinik Dr. Dr. h. c. H.-J. Koch

Am Schönenwald

55765 Birkenfeld

www.t-klinik.de 